\title{
Studying an engineering model on an air blast wave
}

\author{
Z. Wang, X. Gong, J. Xiong \& H. Yong \\ Institute of Applied Physics and Computational Mathematics, \\ Beijing, China
}

\begin{abstract}
This paper presents a new engineering model on the air blast wave of TNT explosive. The model is built and the parameters are calibrated based on numerical simulated pressure time data of shock wave in air. The model describes the peak overpressure, impulse, typical time and attenuation coefficient of the shock wave with new computational equations. Comparison of pressure configuration for the scaled distance shows that the present engineering model can give consistent results with the numerical code and experiments.
\end{abstract}

Keywords: blast wave, overpressure, numerical simulation, engineering model.

\section{Introduction}

Impact loading of air blast is important in a variety of weapon design and the research of defending safeguard. The engineering computational model of air blast wave describes the rules of overpressure time with respect to the scaled distance after an explosion in air of equivalent TNT explosive. Based on analysis of experiments Friedlander [1] presented a strong practical model that the overpressure distribution in time at the positive phase zone is $\Delta p^{+}(t)=\Delta p_{s}(1-\bar{t}) e^{-\alpha \bar{t}}$, where $\Delta p_{s}$ is peak overpressure, $\bar{t}$ is dimensionless time that determines the time when shock front arrives and the positive phase duration, $\alpha$ is the attenuation coefficient of overpressure. $\Delta p_{s}, \bar{t}$ and $\alpha$ are crucial in importance for describing overpressure distribution and many researchers persevered in improving the computational model of them [2-10]. As peak overpressure $\Delta p_{s}$, a lot of engineering equations have been presented based on experiments or numerical simulation [2-7]. Among them, Henrych [5] and Kinney and Grahma [6] showed consistent results with test data for nearly 
all the range of the scaled distance. But $\bar{t}$ and $\alpha$ are closely related to evolution of overpressure and impulse of shock wave in air and they showed very complicated nonlinearly for different amount of explosive and scaled distance. So it is very difficult to determine widely adaptive equations for two of them just by analysis of experiments. Another problem is about the negative phase duration of air blast wave which is usually ignored in the previous studies but a number of researchers thought it is very important [8-10].

The motivation of the article is to build a new engineering computational model that can nicely describe air blast wave for a long range of the scaled distance. And the simple and practical equations are tried to be used for the model based on numerical simulated pressure time data. Some results of numerical simulation on explosion process of $1 \mathrm{~kg}$ spherical TNT explosive are given firstly as the base of study, and then the computational approaches for the peak overpressure, minimum negative overpressure, impulse, typical time and attenuation coefficient are presented. After all the parameters are calibrated, some comparison of overpressure distribution shows that the present model gives consistent results with numerical code and experiments.

\section{Numerical simulation results}

A Lagrange code CHAP is used for numerical simulation on explosion process. The EOS for detonation products is Jones-Wilkins-Lee (JWL) as

$$
P=\mathrm{A}\left(1-\frac{\omega}{\mathrm{R}_{1} \bar{v}}\right) \exp \left(-\mathrm{R}_{1} \bar{v}\right)+\mathrm{B}\left(1-\frac{\omega}{\mathrm{R}_{2} \bar{v}}\right) \exp \left(-\mathrm{R}_{2} \bar{v}\right)+\omega \frac{E}{\bar{v}}
$$

where $\bar{v}=v / v_{0}$ is relative volume, $E$ is internal energy per unit reference volume. The others parameters are $\mathrm{A}=373.77 \mathrm{GPa}, \mathrm{B}=3.7471 \mathrm{GPa}, \mathrm{R}_{1}=4.5, \mathrm{R}_{2}$ $=0.9, \omega=0.35$. The density of TNT is $\rho_{T N T}=1.63 \mathrm{~g} / \mathrm{cm}^{3}$, the detonation speed is $D_{\mathrm{CJ}}=6.93 \mathrm{~km} / \mathrm{s}$, the detonation pressure is $p_{\mathrm{CJ}}=21.0 \mathrm{GPa}$, and the initial internal energy per unit reference volume $E_{0}=6.08 \mathrm{GPa}$.

The ideal gas law is used for the EOS of air, including air density $\rho_{0}=1.225 \mathrm{~kg} / \mathrm{cm}^{3}$, and ideal gas constant $\gamma=1.4$.

A schematic of the blast wave based on numerical simulation with CHAP code is shown in Figure 1. The shock wave has an instantaneous rise and an exponential decay process. $T_{a}, T_{r}, T_{d}$ and $T_{f}$ are the shock wave front arrival time, the rising time for peak overpressure, the duration time for the positive overpressure and the final time that minimum negative overpressure can decrease to $T_{a}$ is closely equal to $T_{r}$ and the overpressure time history is usually assumed starting from the peak value and decreases exponentially, so $T_{a}$ is ignored here just as in previous studies.

For a spherical free field explosion of $1 \mathrm{~kg}$ TNT explosive, $T_{r}, T_{d}, T_{f}$, the peak overpressure $\Delta p_{s}$, the minimum negative overpressure $\Delta p_{f}$ and the impulse of positive phase duration $I^{+}$are shown in Table 1 with the distance from the explosion centre. 


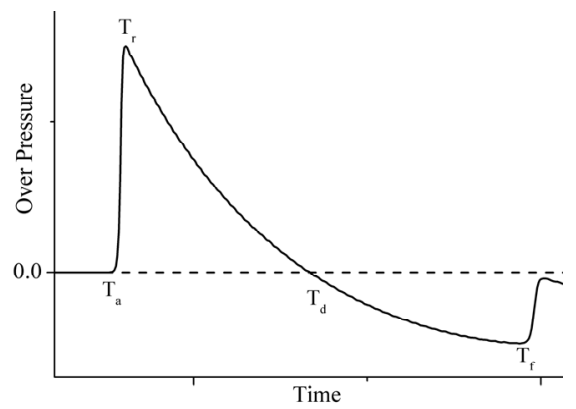

Figure 1: A schematic of the blast wave based on numerical simulation.

Table 1: Overpressure data with the explosion distance (1 kg spherical TNT).

\begin{tabular}{|c|c|c|c|c|c|c|}
\hline$R(\mathrm{~m})$ & $T_{\mathrm{r}}(\mathrm{ms})$ & $T_{\mathrm{d}}(\mathrm{ms})$ & $T_{\mathrm{f}}(\mathrm{ms})$ & $\Delta p_{f}(\mathrm{bar})$ & $\Delta p_{s}(\mathrm{bar})$ & $I^{+}(\mathrm{Pa} \cdot \mathrm{s})$ \\
\hline 0.8 & 0.3610 & 0.9443 & 1.9584 & 0.3726 & 13.4027 & 145.4982 \\
\hline 1.0 & 0.5479 & 1.2095 & 2.2323 & 0.3658 & 8.2580 & 112.8874 \\
\hline 2.0 & 2.0734 & 3.3999 & 5.2379 & 0.2304 & 1.5617 & 67.3324 \\
\hline 4.0 & 6.6863 & 8.9978 & 11.8155 & 0.1119 & 0.4049 & 37.4544 \\
\hline 6.0 & 11.9571 & 14.7891 & 17.8966 & 0.0728 & 0.2107 & 25.3884 \\
\hline 8.0 & 17.4536 & 20.6226 & 23.9346 & 0.0535 & 0.1376 & 19.1324 \\
\hline 10.0 & 23.0598 & 26.4728 & 29.9331 & 0.0422 & 0.1005 & 15.3320 \\
\hline 12.0 & 28.7281 & 32.3318 & 35.9059 & 0.0347 & 0.0784 & 12.7866 \\
\hline 14.0 & 34.4375 & 38.1979 & 41.8715 & 0.0294 & 0.0639 & 10.9635 \\
\hline 16.0 & 40.1745 & 44.0653 & 47.8310 & 0.0256 & 0.0536 & 9.5944 \\
\hline 18.0 & 45.9316 & 49.9358 & 53.7762 & 0.0225 & 0.0461 & 8.5289 \\
\hline 20.0 & 51.7962 & 55.7858 & 59.5719 & 0.0195 & 0.0383 & 7.6353 \\
\hline 22.0 & 57.5850 & 61.6841 & 65.4859 & 0.0176 & 0.0340 & 6.9415 \\
\hline 24.0 & 63.3894 & 67.5199 & 71.3843 & 0.0160 & 0.0305 & 6.3633 \\
\hline 26.0 & 69.1940 & 73.4339 & 77.2515 & 0.0146 & 0.0276 & 5.8734 \\
\hline 28.0 & 74.9985 & 79.2698 & 83.2751 & 0.0136 & 0.0251 & 5.4537 \\
\hline 30.0 & 80.8187 & 85.1682 & 89.1266 & 0.0126 & 0.0231 & 5.0903 \\
\hline 32.0 & 86.6389 & 91.0666 & 95.1189 & 0.0117 & 0.0213 & 4.7717 \\
\hline 34.0 & 92.4748 & 96.8869 & 100.9704 & 0.0110 & 0.0198 & 4.4910 \\
\hline 36.0 & 98.2950 & 102.7853 & 106.9471 & 0.0103 & 0.0185 & 4.2416 \\
\hline 38.0 & 104.1309 & 108.6838 & 112.7674 & 0.0098 & 0.0173 & 4.0182 \\
\hline 40.0 & 109.9668 & 114.5510 & 118.7441 & 0.0092 & 0.0163 & 3.8169 \\
\hline 42.0 & 115.8183 & 120.4025 & 124.5643 & 0.0088 & 0.0153 & 3.6352 \\
\hline 44.0 & 121.6542 & 126.2854 & 130.5254 & 0.0084 & 0.0145 & 3.4702 \\
\hline
\end{tabular}

These data in Table 1 are the base of building the engineering blast model and calibrating parameters. As effected by the interface of detonation products, the schematic of the blast wave when $R<0.8 \mathrm{~m}$ is different from Figure 1. Another note is that about 3000 other data are used for nicely analysis of peak overpressure $\Delta p_{s}$ at the range of $0.07<R<46.40 \mathrm{~m}$. 


\section{Building engineering model}

\subsection{Overpressure and impulse}

The scaled distance is $\bar{R}=R / \sqrt[3]{W}$, where $R$ in $\mathrm{m}$ is the distance to explosion centre, $W$ in $\mathrm{kg}$ is the mass of TNT explosive. The results of air blast wave are usually analyzed in double logarithmic diagrams in literature, so in this article the peak overpressure $\Delta p_{s}$ is described as

$$
\lg \Delta p_{s}=\mathrm{a} \mathrm{e}^{-\mathrm{b} \bar{R}}-\mathrm{c} \lg \bar{R}+\mathrm{d}
$$

where a, b, c and d are parameters. When $\bar{R}$ gets large enough, Eqn (2) can be simplified to $\lg \Delta p_{s} \approx-\mathrm{c} \lg \bar{R}+\mathrm{d}$ which shows linearly relations in double logarithmic diagrams. An exponential decay item is added in Eqn (2) compared to overpressure model of $\mathrm{Wu}$ and Hao [7] at the large scaled distance.

Based on numerical simulation data, all the parameters are fitted and $\Delta p_{s}$ in bar is determined as

$$
\lg \Delta p_{s}=\left\{\begin{array}{lc}
1.135 \mathrm{e}^{-0.677 \bar{R}}-1.318 \lg \bar{R}+0.325, & 0.3 \leq \bar{R} \leq 46.4 \mathrm{~m} / \sqrt[3]{\mathrm{kg}} \\
-2.56 \mathrm{e}^{-5.55 \bar{R}}-3.34 \lg \bar{R}+0.67, & \bar{R}<0.3 \mathrm{~m} / \sqrt[3]{\mathrm{kg}}
\end{array}\right.
$$

Figure $2 \mathrm{~A}$ shows the comparison of the peak overpressure results between numerical simulation and the fitted data and they agree very well. Figure $2 \mathrm{~B}$ is relative error of fitted data for overpressure and it shows the maximum error is about $7 \%$. Figure 3 is the comparison of the peak overpressure between the present model and the previous engineering equations [2-7] and some experiments in literature $[11,12]$.

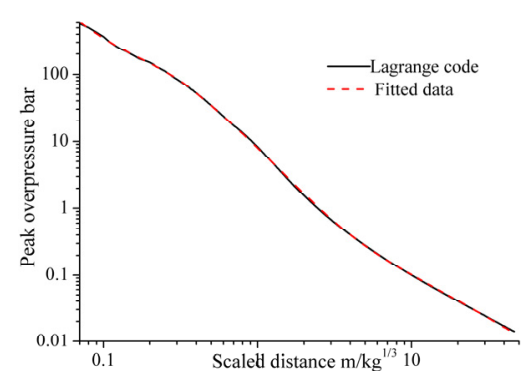

A: peak overpressure

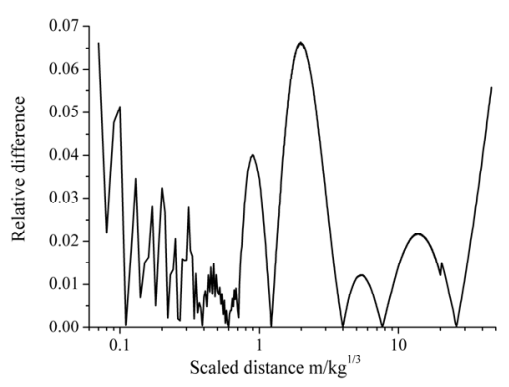

B: relative error of fitted data

Figure 2: Comparison of the peak overpressure. 


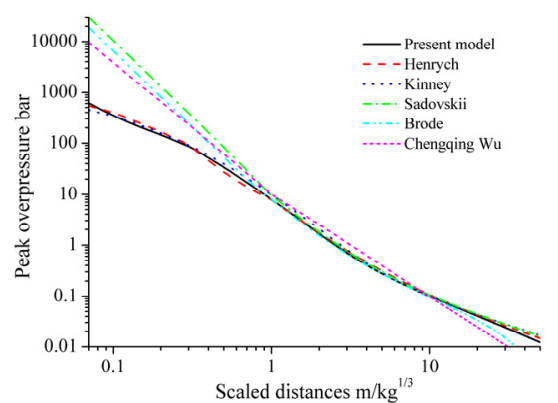

A: with engineering equations

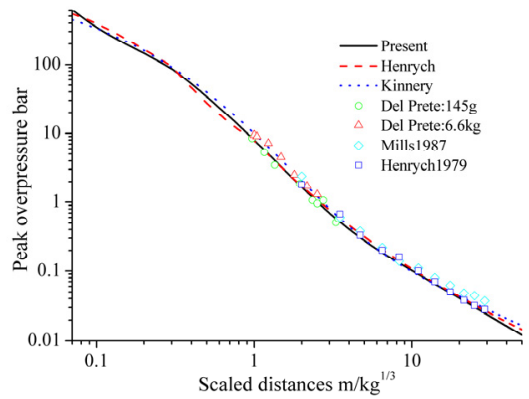

B: with test data in literature $[11,12]$

Figure 3: Comparison of the peak overpressure.

Figure 3A shows that the present model gives consistent results with the equation of Henrych and Kinney and the other equations give greater overpressure when $\bar{R} \leq 1 \mathrm{~m} / \sqrt[3]{\mathrm{kg}}$. Some test data of the overpressure at the large scaled distance are added in Figure 3B.

Eqn (2) is also used to describe the minimum negative overpressure $\Delta p_{f}$ and the positive phase impulse $I^{+}$and after fitted data they are given as

$$
\begin{gathered}
\lg \Delta p_{f}=-1.98 \mathrm{e}^{-2.67 \bar{R}}-1.08 \lg \bar{R}-0.3 \\
\lg I^{+}=-0.24 \mathrm{e}^{-0.7 \bar{R}}-\lg \bar{R}+2.186
\end{gathered}
$$

Eqn (5) is the impulse for $1 \mathrm{~kg}$ TNT, so it should be times $\sqrt[3]{W_{\mathrm{TNT}}}$ for the mass of TNT explosive $W_{\mathrm{TNT}}$. Figures 4 and 5 show that the fitted results $\Delta p_{f}$ and $I^{+}$ agree well with the numerical simulation data and they are linearly changed at a large scaled distance.

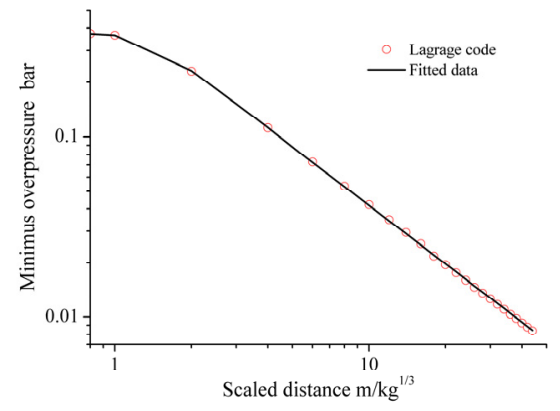

Figure 4: Comparison of $\Delta p_{f}$.

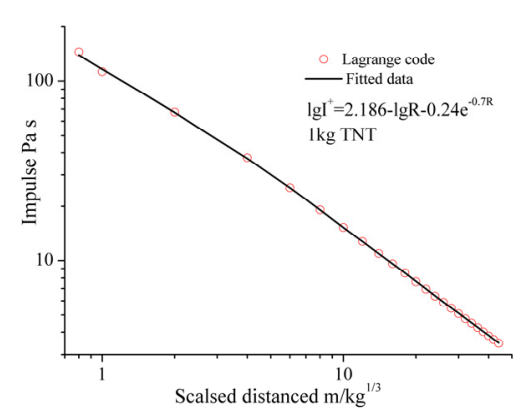

Figure 5: $\quad$ Comparison of $I^{+}$.

\subsection{Typical time}

The model of $T_{r}, T_{d}$ and $T_{f}$ need to be determined for building an engineering model for describing overpressure distribution such as Figure 1. 
$T_{r}$ can be computed by the relation of the shock front speed $D_{s}$ with the peak pressure $p_{s}$ in air as

$$
D_{s}=\sqrt{\frac{(\gamma+1) p_{s}+(\gamma-1) p_{0}}{2 \rho_{0}}}
$$

where $p_{s}=\Delta p_{s}+p_{0}, p_{0}$ is the ambient pressure. So $T_{r}(R)$ can be derived by numerical integration as

$$
T_{r}=T_{0}+\int_{R_{0}}^{R} \frac{1}{D_{s}} d R=T_{0}+\int_{R_{0}}^{R} \sqrt{\frac{2 \rho_{0}}{(\gamma+1) p_{s}(R)+(\gamma-1) p_{0}}} d R
$$

where $R_{0}$ is the radius of the spherical explosive, $T_{0}=R_{0} / D_{\mathrm{CJ}}$ is the time that the detonation wave gets to the outer boundary of the explosive. When $\gamma=1.4$, Eqn (7) can be simplified to

$$
T_{r}=T_{0}+\int_{R_{0}}^{R} \sqrt{\frac{5 \rho_{0}}{6 p_{s}(R)+p_{0}}} d R
$$

$T_{d}$ and $T_{f}$ are assumed to be given by

$$
\begin{aligned}
& T_{d}=\left(1+k_{d} / \bar{R}\right) T_{r} \\
& T_{f}=\left(1+k_{f} / \bar{R}\right) T_{r}
\end{aligned}
$$

where $k_{d}$ and $k_{f}$ are parameters. By fitted data in Table $1, k_{f}=3.1$ is determined and from

$$
\left(T_{f}-T_{d}\right) /\left(T_{d}-T_{r}\right)=k_{f} / k_{d}-1=\mathrm{f}(\overline{\mathrm{R}})
$$

$k_{d}$ is determined by fitted model as

$$
k_{f} / k_{d}-1=0.93+0.89 \exp (-0.28 \overline{\mathrm{R}})
$$

Figure 6 is the results of $f(\bar{R})$ from numerical simulation and Eqn (12). Figure 7 shows the comparison of the typical time $T_{r}, T_{d}$ and $T_{f}$ between numerical simulation and the present model and they agree well at a long range of scaled distance.

\subsection{Attenuation coefficient of overpressure}

When $T_{r} \leq t \leq T_{d}$, the overpressure model is described by Friedlander model as

$$
\Delta p^{+}(t)=\Delta p_{s}(1-\bar{t}) e^{-\alpha \bar{t}}, \bar{t}=\left(t-T_{r}\right) /\left(T_{d}-T_{r}\right)
$$

There are a lot of models such as Henrych [5], Wu and Hao [7] and Borgers and Vantomme [13] ( $\left.\alpha=1.5 \bar{R}^{-0.38}\right)$ to determine the attenuation coefficient $\alpha$. But we cannot obtain good results by using these models of $\alpha$ in the previous literature. 


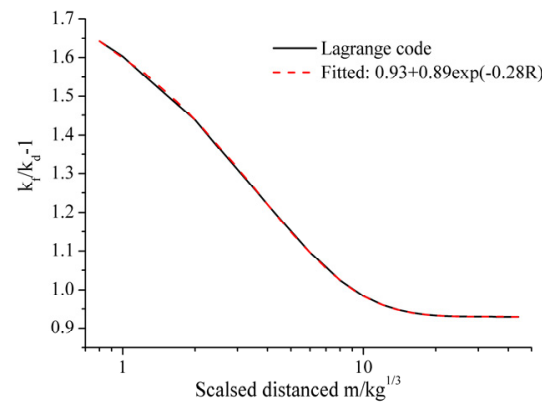

Figure 6: $\quad$ Results of $f(\bar{R})$.

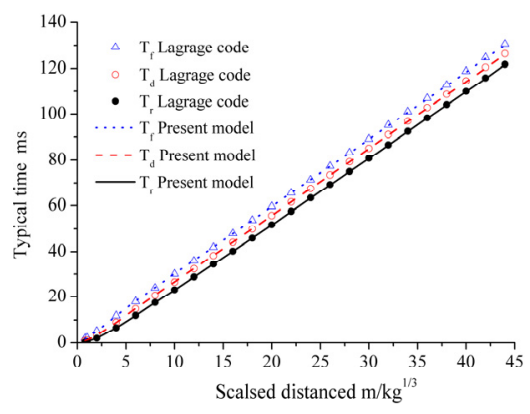

Figure 7: Results of typical time.

If $\alpha$ is not related to time, with Eqn (13) and $\int_{T_{r}}^{T_{d}} \Delta p^{+}(t) d t=I^{+}, \alpha$ can be determined as

$$
\frac{\alpha^{2}}{\alpha-1+e^{-\alpha}}=\frac{\Delta p_{s}\left(T_{d}-T_{r}\right)}{I^{+}}
$$

With the scaled distance increasing, the positive phase overpressure time distribution will be gradually close to a triangle curve, so $\alpha$ will gradually decrease to zero.

In the negative overpressure zone, when $T_{d} \leq t$, Eqn (15) is used to describe the rule of overpressure and time.

$$
\Delta p^{-}(t)=\Delta p^{*}(1-\bar{t}) \mathrm{e}^{-\beta(t) \bar{t}}
$$

where $\beta(t)$ is the attenuation coefficient and it is related to time. $\Delta p^{*}$ is parameter.

Here we note $\beta_{d}=\beta\left(T_{d}\right), \quad \beta_{f}=\beta\left(T_{f}\right), \quad \beta_{f}^{\prime}=\beta^{\prime}\left(T_{f}\right)=d \beta(t) /\left.d t\right|_{t=T_{f}}$. For keeping the curve of overpressure time distribution continuity, with

$$
d \Delta p^{+}(t) /\left.d t\right|_{t=T_{d}}=d \Delta p^{-}(t) /\left.d t\right|_{t=T_{d}}
$$

We obtain

$$
\Delta p_{s} \mathrm{e}^{-\alpha}=\Delta p^{*} \mathrm{e}^{-\beta_{d}}
$$

With $\Delta p_{f}>0$, note $\bar{t}_{f}=\left(T_{f}-T\right)_{r} /\left(T_{d}-T\right)_{r}$, and $\Delta p^{-}\left(T_{f}\right)=-\Delta p_{f}$ we obtain

$$
\bar{t}_{f} \beta_{f}=\ln \left[\frac{\Delta p_{s}}{\Delta p_{f}}\left(\bar{t}_{f}-1\right)\right]-\alpha+\beta_{d}
$$

As

$$
\frac{d \Delta p^{-}(t)}{d t}=-\frac{\Delta p^{*} e^{-\beta \bar{t}}}{\left(T_{d}-T_{r}\right)^{2}}\left[\left(T_{d}-T_{r}\right)-\beta\left(t-T_{d}\right)-\beta^{\prime}\left(t-T_{r}\right)\left(t-T_{d}\right)\right]
$$


Assuming $d \Delta p^{-}(t) /\left.d t\right|_{t=T_{f}}=0$, we obtain

$$
\beta_{f}\left(T_{f}-T_{d}\right)=\left(T_{d}-T_{r}\right)-\beta_{f}^{\prime}\left(T_{f}-T_{r}\right)\left(T_{f}-T_{d}\right)
$$

So $\beta_{d}$ is the key value, if $\beta_{d}$ is known, with Eqn (17), Eqn (18) and Eqn (20), $\Delta p^{*}, \beta_{f}$ and $\beta_{f}^{\prime}$ can be separately determined. If set $\beta_{d}=\alpha$, then $\Delta p^{*}=\Delta p_{s}$, it is difficult to avoid $d^{2} \Delta p^{-}(t) / d t^{2}<0$ in $T_{d} \leq t \leq T_{f}$ at short scaled distance. Based on analysis of numerical simulation data and the character of Eqn (15), we set $\beta_{f}^{\prime} \geq 0$ for $T_{f} \leq t$ that means $\beta(t)$ will increase after that time and we obtain

$$
\beta_{d} \leq \frac{T_{f}-T_{r}}{T_{f}-T_{d}}+\alpha-\ln \left[\frac{\Delta p_{s}}{\Delta p_{f}}\left(\bar{t}_{f}-1\right)\right]=\beta_{d \max }
$$

Then $\beta_{d}$ is determined as $\beta_{d}=\min \left(\alpha, \beta_{d \max }\right)$.

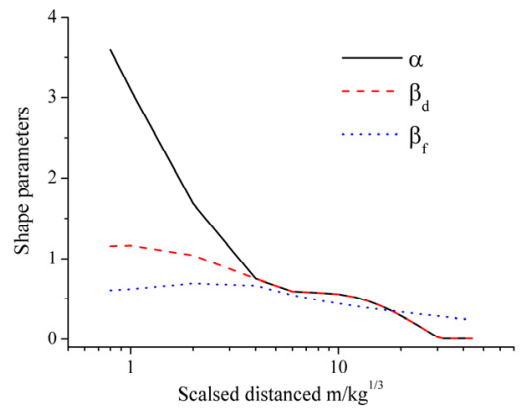

Figure 8: Typical value of attenuation coefficient.

Figure 8 gives the results of $\alpha, \beta_{d}$ and $\beta_{f}$ changing with the scaled distance. Figure 8 shows that $\beta_{d}=\alpha$ is used when it is about $\overline{\mathrm{R}}>4 \mathrm{~m} / \sqrt[3]{\mathrm{kg}}$ and $\beta_{d}$ is limited inside of this zone. When it is about $\overline{\mathrm{R}}>20 \mathrm{~m} / \sqrt[3]{\mathrm{kg}}, \beta_{d}>\beta_{f}$, but as $\beta_{f}^{\prime} \geq 0, \beta(t)$ cannot be set as a simple linear decreasing model in $T_{d} \leq t \leq T_{f}$.

Based on analysis of $\beta_{d}, \beta_{f}$ and $\beta_{f}^{\prime}$, the computational model of attenuation coefficient changing with time in the negative phase duration is assumed as

$$
\beta(t)= \begin{cases}k_{0}+k_{1} t+k_{2} t^{2}, & T_{d} \leq t \leq T_{f} \\ \beta_{f}+\left(t-T_{f}\right) \beta_{f}^{\prime}, & T_{f}<t\end{cases}
$$

where $k_{0}, k_{1}$ and $k_{2}$ are three parameters that can be determined from $\beta_{d}, \beta_{f}$ 
and $\beta_{f}^{\prime}$, such as $k_{2}=\beta_{f}^{\prime} /\left(T_{f}-T_{d}\right)-\left(\beta_{f}-\beta_{d}\right) /\left(T_{f}-T_{d}\right)^{2} \geq 0, k_{1}=\beta_{f}^{\prime}-2 k_{2} T_{f}$, $k_{0}=\beta_{d}-k_{2} T_{d}{ }^{2}-k_{1} T_{d}$. If $\beta_{f} \geq \beta_{d}$, set $k_{2}=0$, it is simple linear changing relation.

When the above models in the negative phase duration are being built, we always insist on two requirements, a) Trying to keep the curve of overpressure time distribution continuity, b) Trying to obtain agreeable results in the zone of $T_{d} \leq t \leq T_{f}$.

\section{Results of the present model}

Up to now, the whole engineering computational model of air blast wave is built and all the parameters can be determined. With a simple code of computing all equations, some results of the model are given by graphs comparing to the results of CHAP code and the results in literature.

Figure 9 shows results of overpressure time history. Good agreement is found between the numerical simulation and the present model. A, B and C in Figure 9 are results of $1 \mathrm{~kg}$ TNT at the explosion distance of 1,14 and $35 \mathrm{~m}$.

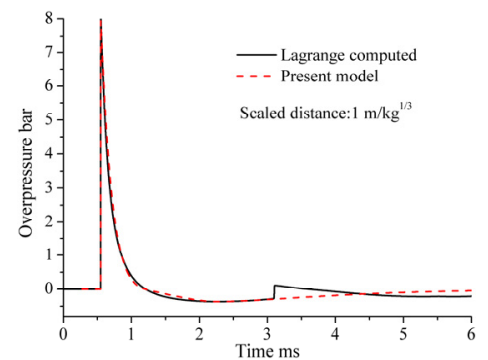

A: $1 \mathrm{~kg}$ TNT $1 \mathrm{~m}$ distance

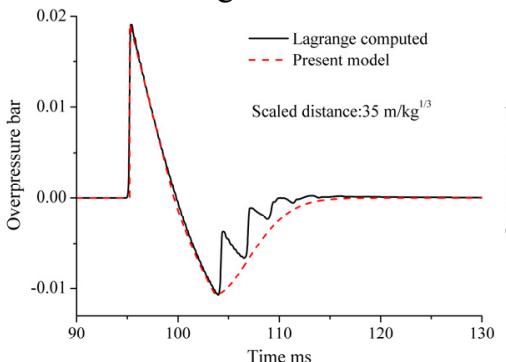

C: $1 \mathrm{~kg}$ TNT $35 \mathrm{~m}$ distance

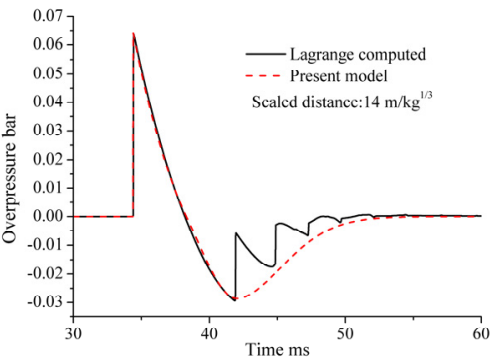

B: $1 \mathrm{~kg}$ TNT $14 \mathrm{~m}$ distance

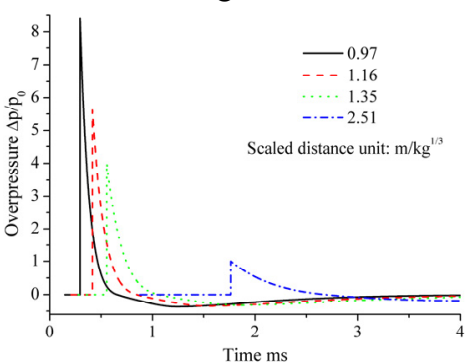

D: $0.18415 \mathrm{~kg}$ TNT

Figure 9: Overpressure time history.

Figure 9D shows the computed results of the present model for $0.18415 \mathrm{~kg}$ TNT at 4 scaled distance as $0.97,1.16,1.35$ and $2.51 \mathrm{~m} / \sqrt[3]{\mathrm{kg}}$. Comparing between results in Figure 9D and Figure 10 which is from literature [11], we can find that the overpressure time distribution are consistent. 

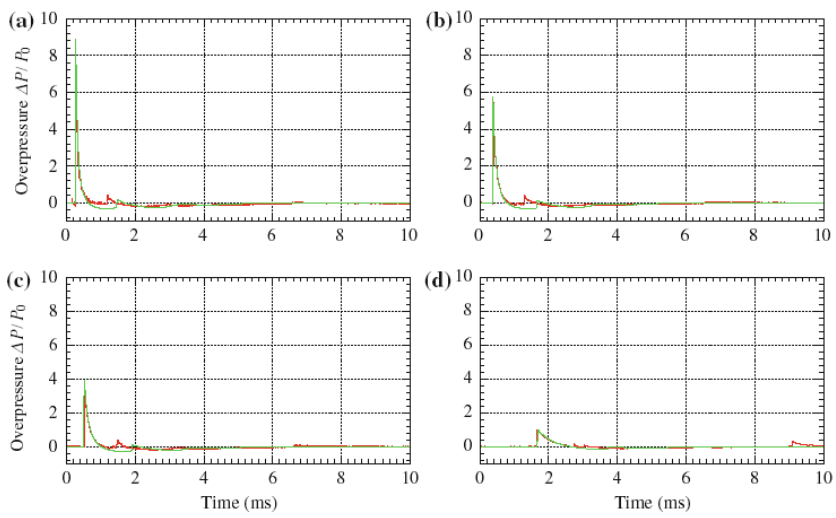

Figure 10: Results of the simulation and experiments in literature [11]. ((a) $0.97 \mathrm{~m} / \sqrt[3]{\mathrm{kg}}$, (b) $1.16 \mathrm{~m} / \sqrt[3]{\mathrm{kg}}$, (c) $1.35 \mathrm{~m} / \sqrt[3]{\mathrm{kg}}$, (d) $2.51 \mathrm{~m} / \sqrt[3]{\mathrm{kg}}$ $0.145 \mathrm{~kg}$ PLANP explosive $(0.18415 \mathrm{~kg}$ equivalent TNT).

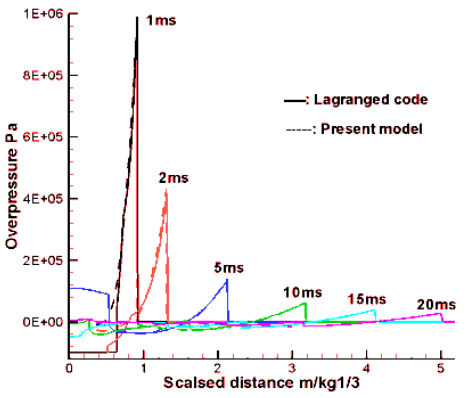

A: $1,2,5,10,15,20 \mathrm{~ms}$

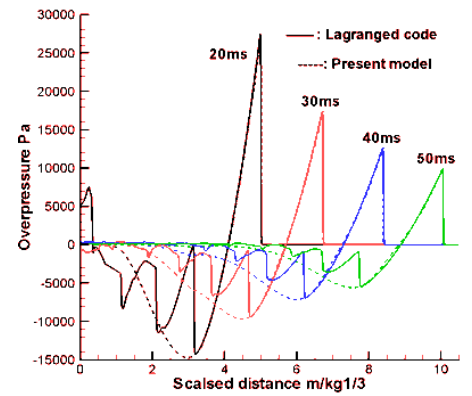

B: $20,30,40,50 \mathrm{~ms}$

Figure 11: $\quad$ Results of overpressure spatial distribution (1 kg TNT).

Figure 11 shows overpressure spatial distribution at different time for $1 \mathrm{~kg}$ TNT explosive and the results between the present model and the numerical simulation agree very well.

\section{Conclusion}

Based on numerical simulated pressure time data, a new engineering computational model of air blast wave of TNT explosive is built and all the parameters can be determined by computing several equations. With the comparison and analysis of overpressure time or spatial distribution, it is showed that the present model can give consistent results with the numerical code and experiments and it can nicely describes air blast wave for a long range of scaled distance. 


\section{Acknowledgements}

The work was performed under the auspices of NSFC (Grant No. 11371065).

The additional support was provided by CAEP under project 2012A0202010.

\section{References}

[1] Friedlander FG, Note on the diffraction of blast waves by a wall. UK Home Office Dept, RC (A), 1939.

[2] Sadovskii MA, Mechanical effects of air shock waves from explosion according to experiments, Moscow: Izd Akad Nauk SSSR, 1952.

[3] Brode HL, Blast Wave from a Spherical Charge. Phys Fluids, 2, 2-17, 1959.

[4] Baker, WE, Cox, PA, Westine, PS, Explosion hazards and evaluations, Elsevier, 1983.

[5] Henrych J, The Dynamics of Explosion and its Use, Amsterdam: Elsevier, 1979.

[6] Kinney GF, Grahma KJ, Explosive shocks in air, New York: Springer, 1985.

[7] Chengqing Wu, Hong Hao, Modeling of simultaneous ground shock and airblast pressure on nearby structures from surface explosions. International Journal of Impact Engineering, 31, 699-717, 2005.

[8] Krauthammer T, Altenberg A, Negative phase blast effects on glass panels. International Journal of Impact Engineering, 24, 1-17, 2000.

[9] Wei J, Mahesh SS, Lokeswarappa RD, Dharani R, Failure analysis of architectural glazing subjected to blast loading. Engineering Failure Analysis. 13, 1029-1043, 2006.

[10] Teich M, Warnstedt P, Gebbeken N, The influence of negative phase loading on cable net facade response. Journal of Architectural Engineering, 18(4), 276-284, 2012.

[11] E·Del Prete, A·Chinnayya, L·Domergue, A·Hadjadj, JF Haas, Blast wave mitigation by dry aqueous foams. Shock Waves, 23, 39-53, 2013.

[12] Adel M·Benselama, Mame JP William Louis, François Monnoyer, A 1D3D mixed method for the numerical simulation of blast waves in confined geometries. Journal of Computational Physics, 228, 6796-6810, 2009.

[13] Borgers JBW, Vantomme J, Towards a parametric model of a planar blast wave created with detonating cord. Proc. of the $19^{\text {th }}$ international symposium on the military aspects of blast and shock, Calgary, Canada; 2006. 